\title{
Sensibilidade gustativa em idosos: uma revisão narrativa
}

\section{Gustative sensitivity in elderly: a narrative review}

\author{
Jamile Ceolin ${ }^{1}$, Thais da Luz Fontoura Pinheiro² \\ 1 Nutricionista. Pós-graduada em Nutrição Clínica com ênfase em Doenças Crônicas e Transtornos Alimentares da Universidade Regional Integrada do \\ Alto Uruguai e das Missões. Frederico Westphalen, RS, Brasil e Mestranda no Programa de Pós-Graduação em Gerontologia Biomédica do Instituto de \\ Geriatria e Gerontologia da Pontifícia Universidade Católica do Rio Grande do Sul. Porto Alegre, RS, Brasil. <jamileceolin@yahoo.com.br> \\ 2 Nutricionista. Mestre em Engenharia de Alimentos. Docente do Curso de Nutrição da Universidade Regional Integrada do Alto Uruguai e das Missões - URI. \\ Frederico Westphalen, RS, Brasil. <thaispinheiro@uri.edu.br>
}

\section{ARTICLE INFO}

\section{Article history}

Received: 15/08/2017

Accepted: 10/11/2017

\section{Correspondent Author}

Jamile Ceolin

Rua Assis Brasil, 709 - Centro

98400-000 Frederico Westphalen, RS, Brasil <jamileceolin@yahoo.com.br>

\section{(c) 2017 All rights reserved}

Editors

Alfredo Cataldo Neto

Paula Engroff

\begin{abstract}
RESUMO
OBJETIVO: Realizar uma revisão narrativa referente ao paladar e os diversos aspectos relacionados às alterações gustativas no envelhecimento, bem como o manejo nutricional. FONTES DE DADOS: Foi realizada uma pesquisa bibliográfica na base de dados PubMed no período de março a julho de 2017. Para execução da busca, utilizaram-se os termos "taste perception", "taste disorders", "taste threshold", "taste bud", "elderly" com auxílio de operadores booleanos (AND e OR). RESUMO DOS ACHADOS: Um dos primeiros receptores envolvidos na identificação e avaliação dos alimentos é o receptor gustativo. Com o envelhecimento, ocorre uma perda de paladar devido a mudanças nas membranas das células gustativas envolvendo função alterada de canais iônicos e receptores sensoriais além da redução do número de botões gustativos numa papila gustativa. Estas desordens podem estar associadas a diversas condições, dentre elas, a polifarmácia, tabagismo, álcool, higiene bucal inadequada e doenças debilitantes como doença renal e hepática, câncer, infecções respiratórias, AIDS e diabetes mellitus, doença de Parkinson e distúrbios cognitivos. Medidas simples como o uso de condimentos e temperos naturais, aumento da ingestão hídrica, mastigação adequada e higiene bucal apropriada podem trazer grandes benefícios ao evitar ou contornar alterações gustativas no idoso, sendo fundamental a atuação do nutricionista. CONCLUSÕES: É essencial compreender as alterações na função gustativa que permeiam o processo de envelhecimento a fim de detectar precocemente essas alterações e contribuir para uma melhor qualidade de vida dos idosos.
\end{abstract}

PALAVRAS-CHAVE: envelhecimento; idoso; paladar; distúrbios do paladar.

\begin{abstract}
OBJECTIVE: This study is a narrative review about the palate and the various aspects related to taste alterations in aging, as well as nutritional management. DATA SOURCES: A bibliographic search was carried out in the PubMed database from March to July 2017. For the purpose of the search, the terms "taste perception", "taste disorders", "taste threshold", "taste" bud", "elderly" with the help of Boolean operators (AND and OR). SUMMARY: One of the earliest recipients involved in food identification and evaluation is the taste receptor. With aging, a loss of taste occurs due to changes in the membranes of taste cells involving altered function of ion channels and sensory receptors in addition to reducing the number of taste pores. These disorders may be associated with several conditions, including polypharmacy, smoking, alcohol, inadequate oral hygiene and debilitating diseases such as kidney and liver disease, cancer, respiratory infections, AIDS and diabetes mellitus, Parkinson's disease and cognitive disorders. Simple measures such as the use of natural condiments and seasonings, increased water intake, proper chewing and proper oral hygiene can bring great benefits by avoiding or circumventing gustatory changes in the elderly, being essential the performance of the nutritionist. CONCLUSIONS: It is essential to understand the changes in gustatory function that permeate the aging process in order to detect these changes early and contribute to a better quality of life for the elderly.

KEYWORDS: aging; elderly; taste; taste disorders.
\end{abstract}




\section{INTRODUÇÃO}

O envelhecimento populacional configura-se atualmente como uma realidade da maioria das sociedades e abarca as mudanças na estrutura etária de uma dada população, produzida pelo aumento relativo de pessoas idosas. ${ }^{1}$ Segundo projeções efetuadas pelo Instituto Brasileiro de Geografia e Estatística, ${ }^{2}$ a população idosa brasileira irá quadruplicar até 2060, representando $26,7 \%$ da população total.

Com o envelhecimento ocorrem alterações fisiológicas e anatômicas que repercutem nas condições de saúde e nutrição do idoso. Essas mudanças progressivas incluem redução da capacidade funcional, alterações de processos metabólicos do organismo e diminuição do paladar, interferindo na sensibilidade para os gostos primários (doce, salgado, ácido e amargo). . $^{3-4}$

Devido ao processo de senescência, é comum a ocorrência de diminuição do paladar. Assim, indivíduos idosos precisam de um estímulo gustativo mais intenso para produzir a mesma sensação de gosto que um indivíduo jovem. Tal deterioração pode ocasionar inapetência, monotonia alimentar, diminuição da ingestão e desnutrição. ${ }^{5}$ Além disso, a redução da função sensorial pode afetar a segurança pessoal e a qualidade de vida, o que pode ter consequências negativas adicionais para a saúde do idoso. ${ }^{6}$

Diversos são os fatores que podem influenciar na redução da função gustativa em idades mais avançadas, dentre eles as comorbidades, as deficiências funcionais e cognitivas, a depressão ou a polifarmácia, os quais foram documentados como contribuintes significativos para distúrbios do paladar em idosos. ${ }^{7}$

Sendo assim, o objetivo deste artigo foi realizar uma revisão narrativa de literatura referente ao paladar e os diversos aspectos relacionados às alterações gustativas próprias do processo fisiológico e dos eventos secundários ao envelhecimento, bem como abordar o manejo nutricional nessa condição clínica.

\section{REVISÃO DE LITERATURA}

O delineamento metodológico deste estudo caracterizou-se por ser uma revisão narrativa, baseada no uso de métodos que visam à busca de um assunto em acervos da literatura.

Foi realizada uma pesquisa bibliográfica na base de dados PubMed no período de março a julho de 2017. Para execução da busca, utilizaram-se os termos "taste perception", "taste disorders", "taste threshold", "taste bud", "elderly" com auxílio de operadores booleanos (AND e OR). Essas expressões foram pesquisadas combinadas entre si ou isoladas. Artigos e livros citados nas referências também foram identificados e analisados. Foram consideradas publicações nos idiomas inglês, português e espanhol.

$\mathrm{Na}$ busca, foram identificadas 135 referências. Após a leitura dos títulos e/ou resumos e as referências bibliográficas dos artigos de interesse, foram incluídos 30 artigos e 6 livros nesta revisão. $\bigcirc$ estudo abrangeu publicações a partir dos anos 2004 até 2017 e livros de 1980 à 2016.

Esta revisão narrativa foi agrupada em cinco categorias: fisiologia do paladar, alterações gustativas no envelhecimento, fatores associados à redução da sensibilidade gustativa, patologias associadas com a redução do paladar e o manejo nutricional nas alterações do paladar.

\section{Fisiologia do paladar}

Um dos primeiros receptores envolvidos na identificação e avaliação dos alimentos é o receptor gustativo. Os receptores do gosto, conhecido como botões ou corpúsculos gustativos, são estruturas epiteliais distribuídas sobre a língua, podendo ocorrer no palato mole, faringe, epiglote e parte superior do esôfago. ${ }^{8}$

O paladar é uma função principalmente dos botões gustativos, mas constata-se comumente que cerca de $90 \%$ do sabor depende do olfato, ressaltando sua relação com a percepção gustativa. ${ }^{9-10} \mathrm{O}$ termo paladar é na realidade uma sensação plurimodal, na qual as sensações de olfato, calor ou frio, pressão ou mesmo de dor, se sobrepõem às sensações gustativas propriamente ditas. ${ }^{11}$ Sua importância reside no fato de permitir ao indivíduo selecionar os alimentos segundo seu desejo e necessidades do organismo. ${ }^{9}$

O sistema gustativo distingue cinco qualidades de estímulos básicos: doce, salgado, ácido (azedo), amargo e umami.

O gosto doce é percebido na ponta da língua, o salgado anteriormente no dorso (base e bordas), o ácido ao longo das bordas e o amargo na parte posterior (base). Os gostos ácido e amargo também podem ser percebidos no palato juntamente com alguma sensibilidade ao doce e ao salgado e todos os tipos de gostos podem ser percebidos na faringe e epiglote. ${ }^{12}$

O gosto umami é percebido quando aminoácidos (como glutamato e aspartato) e nucleotídeos (monofosfatos de inosinato ou guanilato, inosina 5'-monofosfato e guanosina 5'-monofosfato) dos alimentos interagem com as células gustativas. ${ }^{13-14}$ Diferente dos quatro gostos básicos, o umami não exibe sabor extenso mesmo quando a concentração 
de substâncias umami é amplamente aumentada. Este sabor se harmoniza com outros gostos em alimentos e traz brandura e delicioso sabor palatável. ${ }^{15}$

Embora fisiologicamente estimulem mecanismos específicos, os sabores primários, obedecem a sinais químicos relativamente inespecíficos, porque os receptores não são excitados somente por um tipo de estímulo químico, mas por um conjunto de sinais químicos capazes de excitar dito receptor. ${ }^{16}$

No ser humano, a superfície da língua é revestida por uma membrana mucosa, a qual se dobra, em vários lugares, formando pequenas saliências denominadas papilas. ${ }^{11}$ Os botões gustativos estão agrupados nas papilas fungiformes (semelhantes a cogumelos, situadas nos $2 / 3$ anteriores da língua), papilas foliadas (são dobradas, para formar estruturas semelhantes a folhas, na borda posterior da língua) e papilas circunvaladas (estruturas grandes, arredondadas, circundadas por uma goteira, situadas na parte posterior da língua). ${ }^{17}$ Dentro dos corpúsculos distinguem-se quatro tipos de células: basais; de sustentação (tipo 1 e 2) e receptoras gustativas (tipo 3) que fazem as conexões sinápticas com fibras nervosas sensoriais. ${ }^{12}$

Os botões gustativos orais têm como função analisar os constituintes químicos do alimento que entra em contato com as células receptoras durante a mastigação. As substâncias químicas em solução produzem mudanças nas células gustativas que são transformadas em potenciais de ação nas fibras nervosas que inervam os botões gustativos e são conduzidos para o cérebro, produzindo a sensação do gosto. ${ }^{8}$

As fibras gustativas que transmitem as respostas do conjunto dos botões gustativos pertencem a diferentes nervos cranianos. ${ }^{11}$ As fibras dos dois terços anteriores da língua trafegam, primeiro, por ramos do nervo trigêmeo e, depois, pela corda do tímpano, um ramo do nervo facial. A sensação gustação do terço posterior da língua é conduzida por fibras do nervo glossofaríngeo, enquanto outras fibras da epiglote e de outras áreas cursam por ramos do nervo vago. A partir de sua entrada no troco cerebral, todas as fibras gustativas se reúnem formando o trato solitário e, finalmente fazem sinapse na porção rostral do núcleo do mesmo. Desse ponto, os axônios passam por vias mal definidas, para o núcleo ventromedial do tálamo e, depois, para o córtex cerebral, na região central do giro pós-central, que se enrola para dentro da fissura lateral. ${ }^{18}$

Quatro operações são realizadas pelos botões gustativos: detecção, intensidade, reconhecimento e discriminação. A detecção identifica a presença ou ausência do estímulo, a intensidade é a codificação da magnitude do estímulo, o reconhecimento, quando há uma identificação positiva do estímulo e a discriminação é a codificação da informação referente às diferenças entre os estímulos. ${ }^{8}$

Os distúrbios do gosto (disgeusias) podem ser classificados em distúrbios qualitativos e quantitativos. Os qualitativos incluem a parageusia (percepção de gosto inadequada ou errada provocada por um estímulo) e phantogeusia (presença de um sabor persistente e desagradável na ausência de qualquer estímulo). Os quantitativos são ageusia (perda completa da capacidade de provar), hipogeusia (perda parcial da capacidade de provar) e hipergeusia (sensibilidade gustativa aprimorada). ${ }^{19-20}$

\section{Alterações gustativas no envelhecimento}

O envelhecimento natural produz perda de paladar devido a mudanças nas membranas das células gustativas envolvendo função alterada de canais iônicos e receptores sensoriais. ${ }^{21}$ Além disso, há uma grande redução do número de botões gustativos numa papila gustativa, o que ocasiona alterações no limiar gustativo tanto por avaliação qualitativa como por avaliação eletrofisiológica. ${ }^{12}$

O gosto tem base anatômica no número de corpúsculos gustativos nas papilas linguais. Em pessoas jovens, esse número corresponde a mais de 250 corpúsculos para cada papila, enquanto nas pessoas acima de 70 anos esse valor é menor que 100 papilas gustativas linguais, o que acarreta um decréscimo do limiar de detecção e identificação de sabor em consequência do envelhecimento. Sendo assim, o idoso necessita de maior concentração de elementos indutores da sensação de sabor na constituição dos alimentos em comparação aos adultos jovens. ${ }^{22}$

As perdas quimiosensoriais tornam-se evidentes a partir dos 60 anos aumentando severamente após os 70 anos prejudicando a saúde global, e por consequência influenciando na qualidade de vida dos idosos. ${ }^{23}$

Distúrbios do paladar são frequentemente subestimados, mas podem ter uma precipitação desfavorável sobre a saúde das pessoas idosas, tais como, inapetência, mudanças nas preferências alimentares, refeições monótonas, anorexia, perda de peso e desnutrição, tendo como consequência o agravo das suas doenças crônicas. $^{24}$ Dessa forma, a acuidade do paladar é importante, pois permite não somente avaliar o valor nutricional da dieta, a segurança e a qualidade dos alimentos e líquidos, mas também proporciona um dos maiores prazeres da vida, que é o ato de se alimentar. ${ }^{25-26}$

A capacidade reduzida para diferenciar entre várias intensidades ou concentrações de um saborizante pode resultar em aumento da ingestão de sal e açúcar e exacerbação de doenças crônicas, como insuficiência cardíaca, diabetes mellitus e hipertensão. ${ }^{20}$ 
A função do paladar permanece inalterada até a quinta década da vida, em seguida, tende a declinar. A maioria dos estudos indica que o envelhecimento está associado a um decréscimo geral na sensibilidade gustativa que é mais severa no gênero masculino. Em comparação aos indivíduos de 17-18 anos de idade, os idosos de 60-80 anos de idade têm limites maiores, sendo $50 \%$ para a amargura, $25 \%$ para salinidade, $10 \%$ para acidez e $5 \%$ para a doçura. ${ }^{27}$

Ao invés da disfunção gustativa ser em toda boca, os défices regionais na língua são muito mais comuns. Apesar da alta prevalência, a maioria dos idosos não tem conhecimento dessas alterações gustativas. ${ }^{21}$

Em um estudo de revisão sistemática realizado por Methven e colaboradores ${ }^{28}$ concluiu-se que há em geral uma diminuição dos limiares de sabor e sensibilidade gustativa quando relacionado com a idade. No entanto, a extensão do significado deste declínio varia entre modalidades de sabor, saborizantes utilizados e tipos de estudos realizados. O efeito da idade na percepção sensorial e, especificamente, na percepção do paladar, é complexa, devido à grande heterogeneidade da população idosa.

\section{Fatores associados à redução da sensibilidade gustativa}

Fatores associados à perda de paladar incluem, além do envelhecimento normal, o tabagismo, uso de álcool, drogas e as lesões da cavidade oral. A perda de paladar pode resultar na deficiência de zinco e é comum no decurso de várias doenças debilitantes, tais como insuficiência renal aguda e doenças hepáticas crônicas, câncer, síndrome da imunodeficiência adquirida e diabetes mellitus. ${ }^{29}$

Idosos com várias doenças crônicas e que fazem uso de diversos fármacos estão particularmente em risco de alterações do paladar ou perda grave da capacidade de identificar gostos doce, salgado, amargo e ácido. ${ }^{27}$ Os medicamentos que causam alterações do paladar incluem hipoglicemiantes (fenformina e glipizida), antiparkinsonianos (levodopa), anticoagulantes (fenindiona), anti-histamínicos (clorfeniramina), psicoativos (lítio, trifluroperazina) e salicilatos. ${ }^{30}$

Em relação às lesões da cavidade oral, muitos idosos fazem o uso de próteses parciais e totais que por vezes estão mal adaptadas, o que pode causar trauma mecânico e lesionar a gengiva durante o processo de mastigação, podendo interferir no paladar. ${ }^{27}$

Além disso, o envelhecimento é associado com muitas mudanças fisiológicas da cavidade oral. A mucosa oral torna-se mais fina e mais seca devido a uma diminuição da queratinização e um afinamento da estrutura epitelial. Além disso, as glândulas salivares tornam-se gradualmente atrófica e/ou os ácinos desaparecem sendo substituído por tecido conjuntivo fibroso. A densidade do paladar diminui, podendo afetar os diferentes tipos de papilas. ${ }^{27}$

A alteração nas glândulas salivares pode provocar xerostomia e diminuição na produção da amilase salivar, dificultando a digestão oral e a deglutição do bolo alimentar. ${ }^{31} \mathrm{~A}$ dificuldade na digestão inicial dos carboidratos contribui para uma menor percepção do sabor doce, podendo contribuir para o surgimento de alterações glicêmicas em indivíduos que ingerirem carboidratos simples em excesso. ${ }^{22}$

O tabagismo pode afetar a acuidade do gosto. Os resultados de um estudo mostraram que os fumantes aumentaram os limiares de eletrogustometria e diminuíram a vascularização e a densidade de papilas fungiformes em comparação com os indivíduos não fumantes. ${ }^{32}$

A deficiência grave de zinco também pode levar à perda de acuidade do paladar. ${ }^{25}$ Conforme Aliani e colaboradores, ${ }^{33}$ o zinco parece desempenhar um papel na acuidade e deficiência do paladar e é a causa subjacente de certas condições médicas. No entanto, a suplementação de zinco para restaurar a acuidade do paladar mostrou resultados conflitantes em relação a sua eficácia, podendo ser afetada pelas diferentes formas de zinco administradas, as doses utilizadas, a biodisponibilidade e as causas da percepção de sabor alterada.

Em um estudo realizado por Ogawa e colaboradores ${ }^{34}$ foi relatado novos achados de um modelo de estudo longitudinal que teve como objetivo elucidar o declínio da sensibilidade ao gosto e seus fatores relacionados em indivíduos idosos. A amostra foi constituída por 328 sujeitos com idade entre 79-81 anos. De acordo com os resultados obtidos, os homens apresentaram declínios significativamente maiores na sensibilidade do sabor aos gostos doces e amargos do que as mulheres. Além disso, os sujeitos com escores cognitivos mais baixos mostraram uma diminuição do sabor significativamente maior para o salgado. Os autores concluíram que o gênero e o estado cognitivo são os principais fatores que afetam a sensibilidade do sabor em indivíduos geriátricos.

\section{Patologias associadas com alterações do paladar}

Diversas são as condições clínicas crônicas que contribuem para a diminuição da sensibilidade gustativa, dentre elas, a sinusite, as infecções respiratórias, a hepatite $\mathrm{C}$, a doença renal, o diabetes mellitus, as doenças cardíacas, o hipotireoidismo, a AIDS, os distúrbios cognitivos, a doença de Parkinson, 
os problemas dentários (doenças periodontais, cáries), etc. ${ }^{20}$

Pacientes diabéticos parecem ser um grupo propenso a desenvolver um comprometimento na sensibilidade gustativa, principalmente para o gosto doce, quando comparado a outros sabores. Essa diminuição do paladar tem sido associada à hiperglicemia com a presença de uma correlação significativa entre os limiares do paladar e do nível de concentração de glicose no sangue. ${ }^{35}$

Em um estudo realizado por Khobragade, Wakode e Kale, ${ }^{36}$ foi avaliado a relação entre o limiar de sabor em diabéticos tipo 1 e não diabéticos para quatro modalidades básicas de sabor (doce, sal, ácido e amargo). A amostra foi constituída por 70 casos de diabetes mellitus tipo 1 e 70 não diabéticos. Foi observado um aumento significativo no limiar de sabor doce, salgado, ácido e amargo nos pacientes com diabetes mellitus tipo 1 .

Somekawa e colaboradores ${ }^{37}$ avaliaram a relação entre a fragilidade e o paladar e a percepção do olfato de 768 idosos, com 65 anos ou mais, que viviam em áreas urbanas. A baixa percepção do gosto e do cheiro, em particular a percepção do gosto, foi associada a um maior risco de fragilidade em idosos que vivem na comunidade. Através desses resultados, sugere-se que o menor gosto e a percepção do cheiro podem ser um indicador de fragilidade na velhice.

Em uma pesquisa realizada por Kenway e colaboradores, ${ }^{38}$ foi examinado o efeito da idade e a presença de doença (insuficiência renal e câncer) no limiar de sabor. Os resultados do estudo mostraram que o limiar de gosto aumentou com a idade. Nenhuma anormalidade no sabor foi observada em pacientes com insuficiência renal crônica e com câncer. No entanto, ocorreu um sabor alterado em um grande número desses pacientes.

Em um estudo transversal foi investigado se a capacidade do paladar é reduzida em idosos com hospitalização aguda, em comparação com um grupo controle de indivíduos saudáveis com idade pareada. Observou-se que as pessoas idosas hospitalizadas tiveram uma capacidade do paladar significativamente reduzida em comparação com o grupo controle. As qualidades do sabor azedo e amargo foram principalmente afetadas. ${ }^{39}$

Solemdal e colaboradores ${ }^{40}$ investigaram as variáveis de saúde bucal que estão associadas à capacidade de gosto em idosos com hospitalização aguda. Os resultados da pesquisa mostraram que a capacidade do paladar foi reduzida em idosos com hospitalização aguda com presença de cárie, alto crescimento de bactérias orais, má higiene bucal e boca seca. O sabor doce e salgado foram particularmente prejudicados em pacientes com boca seca e o sabor ácido nos indivíduos com alto crescimento de bactérias orais.

Toffanello e colaboradores ${ }^{7}$ investigaram a percepção do paladar em idosos hospitalizados e compararam seus limiares de reconhecimento do gosto com os indivíduos idosos saudáveis e identificaram potenciais determinantes da perda de sabor. Em comparação com os indivíduos saudáveis, os idosos que estavam no hospital foram significativamente menos capazes de reconhecer o sabor do ácido cítrico, sendo associado de forma independente com idade avançada, polifarmácia e mau estado nutricional.

Outro estudo avaliou a percepção gustativa em idosos para os gostos básicos, doce e salgado, em comparação com adultos jovens. Os idosos avaliados precisaram de aproximadamente oito vezes mais sal (cloreto de sódio) do que os adultos para detectar a presença deste componente e de aproximadamente quatro vezes mais açúcar do que os adultos para detectar a presença desta substância. ${ }^{41}$

\section{Manejo nutricional nas alterações do paladar}

A percepção do paladar alterada tem efeitos profundos sobre o estado nutricional, interferindo na qualidade de vida e no aumento da morbidade e mortalidade devido a uma associação com apetite reduzido, inadequação de energia e nutrientes, perda de peso, desnutrição, imunidade reduzida, diminuição da capacidade ou desejo de adquirir alimentos, diminuição da apreciação de alimentos, relações alteradas de alimentos, padrões de ingestão alimentar alterados, rituais e atividades sociais ligados ao comer e beber, angústia emocional e interferência com a vida diária. ${ }^{42}$

As alterações da sensibilidade gustativa que permeiam o processo de envelhecimento impactam consideravelmente sobre o estado nutricional do idoso. Contudo, medidas simples podem trazer grande benefício ao evitar ou contornar alterações gustativas no idoso, dentre elas, a introdução de alimentos que estimulam o paladar (condimentos e temperos naturais), o aumento da ingestão hídrica, a redução do consumo de alimentos com alto teor de carboidratos, em especial os industrializados, estimular a mastigação adequada, higiene bucal apropriada e próteses dentárias em boas condições de uso. ${ }^{22}$

Além disso, a mastigação de goma ou de gelo pode atuar como uma ajuda temporária em indivíduos com alterações de gosto. Os pacientes devem ser encorajados a mastigar seus alimentos muito bem, alternando os lados de sua boca. ${ }^{18}$

Hábitos como esses podem contribuir para um envelhecimento mais saudável, uma vez que esta 
abordagem pode compensar as perdas, minimizando desconfortos e melhorando a qualidade de vida do idoso para além da potencialização da sensibilidade gustativa. 22

Neste sentido, destaca-se o papel do nutricionista, uma vez que este profissional poderá ficar atento a essas alterações durante a avaliação clínica, orientando os pacientes frente a essas questões e intervindo com uma conduta nutricional adequada afim de que o idoso possa despertar o prazer relacionado à comida.

\section{CONSIDERAÇÕES FINAIS}

A diminuição da sensibilidade gustativa é comum em idosos embora sejam frequentemente subestimadas. Sendo a percepção do sabor um fator importante relacionado à qualidade de vida dos idosos com implicações na segurança e adequação nutricional nesses indivíduos, é essencial compreender as alterações na função gustativa que permeiam o processo de envelhecimento a fim de detectar precocemente esses distúrbios.

Através de uma nutrição adequada, é possível aprimorar o sabor dos alimentos, retardar a progressão dessas alterações e atenuar a severidade dos sintomas, contribuindo para um envelhecimento mais saudável e prazeroso. Dessa forma, mais estudos são necessários acerca desta complexa discussão que envolve a percepção do paladar e o manejo nutricional nessas condições clínicas.

\section{REFERÊNCIAS}

1. Cruz DT, Caetano VC, Leite ICG. Envelhecimento populacional e bases legais da atenção à saúde do idoso. Cad. Saúde Colet. 2010;18(4):500-8.

2. Instituto Brasileiro de Geografia e Estatística - IBGE. Projeção da população do Brasil por sexo e idade para o período 2000/2060. [Internet]. Rio de Janeiro; 2013. [Acesso em 2017, 12 de junho]. Disponível em: http:// www.ibge.gov.br/home/estatistica/populacao/projecao_da populacao/2013/default.shtm

3. Campos MTFS, Monteiro JBR, Ornelas APRC. Fatores que afetam o consumo alimentar e a nutrição do idoso. Rev Nutr. 2000;13(3):157-65.

4. Pfrimer K, Ferriolli E. Avaliação Nutricional do Idoso. In: Vitolo MR, org. Nutrição da gestação ao envelhecimento. Rio de Janeiro: RUBIO; 2008. p. 435-49.

5. Alvez LMT, Dantas R. O. Percepção de sabores em pessoas normais. GED gastroenterol Endosc Dig. 2014;33(3):102-5.

6. Schumm LP, McClintock M, Williams S, et al. Assessment of Sensory Function in the National Social Life, Health, and Aging Project. J Gerontol B Psychol Sci Soc Sci. 2009;64(Suppl 1):i76-85.

7. Toffanello ED, Inelmen EM, Imoscopi A, et al. Taste loss in hospitalized multimorbid elderly subjects. Clin Interv Aging. 2013;8:167-74.
8. Bradley RM. Fisiologia Oral Básica. São Paulo: Médica Panamericana; 1981. p. 29-45.

9. Guyton AC. Fisiologia humana. 5aㅡ ed. Rio de Janeiro: Interamericana; 1981. p. 324-6.

10. Landis BN, Beutner D, Frasnelli J, et al. Gustatory function in chronic inflammatory middle ear disease. Laryngoscope. 2005;115:1124-7.

11. Altner H. Fisiologia da gustação. In: Schmidt RF, org. Fisiologia Sensorial. São Paulo, Springer: 1980. p. 264-5.

12. Monteiro MAM. Percepção Sensorial dos Alimentos em Idosos. Revista Espaço para a Saúde. 2009;10(2):34-42.

13. Torii K, Moriyama Y. Taste and Health: Nutritional and Physiological Significance of Taste Substances in Daily Foods Biol. Pharm Bull. 2010;33(11):1771.

14. Singh PB. Cerebral processing of umami: A pilot study on the effects of familiarity. Brain Res. 2015;1614:67-74.

15. Kurihara K. Umami the Fifth Basic Taste: History of Studies on Receptor Mechanisms and Role as a Food Flavor. BioMed Research International. 2015;(2015):1-10.

16. Douglas CR. Fisiologia da Gustação. In: Douglas CR. Tratado de fisiologia aplicada às ciências médicas. Rio de Janeiro: Guanabara; 2006. p. 138-51.

17. Davies A, Blakeley AGH, Kidd C. Paladar e Olfato. In: Davies A, Blakeley AGH, Kidd C. Fisiologia Humana. Porto Alegre: ARTMED; 2002. p. 339-44.

18. Palheta Neto FX, Targino MN, Peixoto VS, et al. Anormalidades sensoriais: Olfato e paladar. Arquivos Int. Otorrinolaringol. 2011;15(3):350-358.

19. Maheswaran T, Abikshyeet P, Sitra G, et al. Gustatory dysfunction. J Pharm Bioallied Sci. 2014;6(Suppl. 1):S30-S33.

20. Syed Q, Hendler KT, Koncilja K. The Impact of Aging and Medical Status on Dysgeusia. Am J Med. 2016;129(7):753. e1-6.

21. Boyce JM, Shone GR. Effects of ageing on smell and taste. Postgrad Med J. 2006;82(966):239-41.

22. Paula RS, Colares FCJ, Toledo JO, et al. Alterações gustativas no envelhecimento. Revista Kairós. 2008;11(1):217-35.

23. Coelho HDS, Granato L. Avaliação dos limiares de detecção do gosto salgado em idosos. J Health Sci Inst. 2014;32(4):413-8.

24. Neumann L, Schauren BC, Adami FS. Sensibilidade gustativa de adultos e idosos. Rev Bras Geriatr Gerontol. 2016;19(5):797-808.

25. França AP, Pivi GAK. Alterações orgânicas, fisiológicas e metabólicas do processo de envelhecimento e seus reflexos na nutrição do idoso. Em: Silva MLN, Marucci MFN, Roediger MA. Tratado de nutrição em gerontologia. Barueri: MANOLE; 2016. p. 49-61.

26. Ogawa T, Annear MJ, Ikebe K, et al. Taste-related sensations in old age. J Oral Rehabil. 2017;44:626-35.

27. Imoscopi A, et al. Taste loss in the elderly: epidemiology, causes and consequences. Aging Clinical and Experimental Research. 2012;24(6):570-9.

28. Methven L, Inelmen EM, Sergi G, et al. Ageing and taste. Proc Nutr Soc. 2012;71:556-65.

29. Kettaneth A, Pariès J, Stirnemann J, et al. Clinical and biological features associates with taste loss in internal medicine patients. A cross-sectional study off 100 cases. Appetite. 2005;44(2):163-9.

30. Yen PK. Nutrition and sensory loss. Geriatr Nurs. 2004; 25(2):118-9. 
31. Strapasson GC, Barreira SMW, Wille GMFC, et al. Fatores que alteram a percepção de sabor. Infarma. 2013;25(2): 111-5.

32. Pavlidis P, Gouveris C, Kekes G, et al. Changes in electrogustometry thresholds, tongue tip vascularization, density and form of the fungiform papillae in smokers. Eur Arch Otorhinolaryngol. 2014;271(8):2325-31.

33. Aliani M, Udenigwe CC, Girgih AT, et al. Zinc Deficiency and Taste Perception in the Elderly. Crit Rev Food Sci Nutr. 2013;53(3):245-250.

34. Ogawa T, Uota M, Ikebe $\mathrm{k}$, et al. Longitudinal study of factors affecting taste sense decline in old-old individuals. J Oral Rehabil. 2017;44(1):22-29.

35. Wasalathanthri S, Hettiarachchi P, Prathapan S. Sweet taste sensitivity in pre-diabetics, diabetics and normoglycemic controls: a comparative cross sectional study. BMC Endocr Disord. 2014;14(67):1-7.

36. Khobragade RS, Wakode SL, Kale AH. Physiological taste threshold in type 1 diabetes mellitus. Indian J Physiol Pharmacol. 2012;56(1):42-7.
37. Somekawa S, Mine T, Ono K, et al. Relationship between Sensory Perception and Frailty in a Community-Dwelling Elderly Population. J Nutr Health Aging. 2017;21(6): $710-4$.

38. Kenway NG, Woo J, Kwan M, et al. Effect of age and disease on taste perception. J Pain Symptom Manage. 2004;28(1):28-34.

39. Solemdal K, Sandvik L, Willumsen T, et al. Taste ability in hospitalised older people compared with healthy, agematched controls. Gerodontology. 2014;31(1):42-8.

40. Solemdal K, Sandvik L, Willumsen T, et al. The impact of oral health on taste ability in acutely hospitalized elderly. PLoS One. 2012;7(5):e36557.

41. Passos JG, Guimarães LC, Victoria MCM. Avaliação da percepção gustativa em idosos para os gostos básicos, doce e salgado, em comparação a jovens adultos. J Health Sci Inst. 2016;34(1):29-32.

42. Boltong A, Keast R. The influence of chemotherapy on taste perception and food hedonics: A systematic review. Cancer Treat Rev.2012;38(2):152-63. 